\title{
Species Segregation and Dynamical Instability of Horizontally Vibrated Granular Mixtures
}

\author{
Massimo Pica Ciamarra, Alessandro Sarracino, Mario Nicodemi, and \\ Antonio Coniglio \\ Dip.to di Scienze Fisiche, Università di Napoli "Federico II" \\ INFM-Coherentia, INFN and AMRA, Napoli, Italy
}

Summary. We review recent results about the segregation process of a granular mixture of disks on an horizontally oscillating tray. In this condition an initially disordered mixture first segregates via the formation of stripes perpendicular to the driving direction; then stripes merge in a coarsening process. We discuss quantitatively both the short-time and the long-time dynamics of the system, and the dependence of the observed phenomenology on the frequency and amplitude of oscillation of the tray. The same system is also investigated when, instead of being disordered, is initially prepared in two stripes parallel to the driving direction. In this condition the interface between the two stripes manifests an instability which again leads to the formation of stripes perpendicular to the driving direction. Finally, we shortly review the mechanism which have been proposed in order to explain the observed segregation process.

\section{Introduction}

A granular medium consisting of a collection of dry, cohesionless, identical particles exhibits a wide range of complex behaviours. Despite the simplicity of the constituent particles no reliable mathematical model exist for most of these collective phenomena. Of particular interest is the counter-intuitive phenomenon of species segregation [1]. When subject to an external perturbation, such as vertical or horizontal oscillations, an initially disordered binary mixture of grains (which may differ in size, mass, frictional properties) often segregates their components. Depending on the driving conditions different mechanism, such as percolation [2-4], inertia [5], convection [6], or even purely thermodynamical effects [7], have been shown to be responsible for the segregation process.

Recently segregation of a binary mixture subject to horizontal oscillations has been observed by T. Mullin and co-workers [8-11]. In their experiment a granular disordered monolayer, composed of a mixture of steel spheres and poppy seeds, where placed on a horizontal tray oscillating along the $x$-axis. 
They observed the system to segregate via the formation of a pattern of alternating stripes of particles of the same kind, parallel to the $y$-axis.

In order to understand the physical mechanism which is responsible for this segregation process we have recently investigated this experiment via soft-core molecular dynamics simulations. Here we review our work [12-14] and present new results relative to the coarsening process of the stripes.

\section{Model}

We perform soft core Molecular Dynamics simulations of a two-dimensional granular media taking into account grain-grain and grain-tray interactions [15]. Two grains with diameters $D_{i}$ and $D_{j}$ in positions $\mathbf{r}_{i}$ and $\mathbf{r}_{j}$ interact if overlapping, i.e., if $\delta_{i j}=\left[\left(D_{i}+D_{j}\right) / 2-\left|\mathbf{r}_{i}-\mathbf{r}_{j}\right|\right]>0$. The interaction is given by a normal repulsive force with viscous dissipation $[16,17]$. In two dimensions this reduces to the linear spring-dashpot model,

$$
\mathbf{f}_{n}=k_{n} \delta_{i j} \mathbf{n}_{i j}-\gamma_{n} m_{\mathrm{eff}} \mathbf{v}_{n i j},
$$

where $k_{n}$ and $\gamma_{n}$ are the elastic and viscoelastic constants, and $m_{\text {eff }}=$ $m_{i} m_{j} /\left(m_{i}+m_{j}\right)$ is the effective mass. We follow the realistic simulations of $[11,15]$ and model the interaction with the tray via a viscous force

$$
\mathbf{f}_{t}=-\mu_{i}\left(\mathbf{v}_{i}-\mathbf{v}_{\text {tray }}\right)
$$

where $\mathbf{v}_{\text {tray }}(t)=2 \pi A \nu \sin (\nu t) \mathbf{x}$ is the velocity of the tray and $\mathbf{v}_{i}$ the velocity of the disk $i$, plus a white noise force $\xi(t)$ with

$$
\left\langle\xi(t) \xi\left(t^{\prime}\right)\right\rangle=2 \Gamma \delta\left(t-t^{\prime}\right) .
$$

For the grain-grain interaction, we use the value $k_{n}=210^{5} \mathrm{~g} \mathrm{~cm}^{2} \mathrm{~s}^{-2}$ and $\gamma_{n}$ chosen, for each kind of grains, such that the restitution coefficient is given: $e=0.8$ [17]. The two components of our mixture have mass $M_{h}=1$ $\mathrm{g}$ and $M_{l}=0.03 \mathrm{~g}$, and viscous coefficient $\mu_{h}=0.28 \mathrm{~g} \mathrm{~s}^{-1}$ and $\mu_{l}=0.34$ $\mathrm{g} \mathrm{s}^{-1}$. The white noise has $\Gamma=0.2 g^{2} \mathrm{~cm}^{2} \mathrm{~s}^{-3}$. Apart from a simple rescaling of masses and lengths, these values are those of reference [11] (and given in private communications), and are taken from direct measurements on the experimental system. We solve the equations of motion by the Verlet algorithm with an integration time-step $d t=6 \mu \mathrm{s}$, which is limited by the value of $K_{n}$ and not, as usual in numerical simulations of the Langevin equation, by the relaxation time $m / \mu$ (in our case $\left.d t \ll M_{l} / \mu_{l} \ll M_{h} / \mu_{h}\right)$. The numerical resolution of stochastic force may be difficult [18]. We have validated the algorithm and the integration time step considering that smaller value of $d t$ reproduces the same results, and that the expected properties of a thermal binary mixture are recovered if the grain-grain interaction is made elastic (i.e. $\gamma_{n}$ is set to zero). 
The heavier grains of our mixture have diameter $D_{h}=1 \mathrm{~cm}$. We consider lighter grains diameter $D_{l}$ to be Gaussian distributed with mean value 0.7 $\mathrm{cm}$ and $17 \%$ polydispersity, or to have the same size of the heavier grains, $D_{l}=D_{h}=1 \mathrm{~cm}$. We use a tray of width $d_{y}=20 \mathrm{~cm}$ and length $d_{x}=40 \mathrm{~cm}$ or $d_{x}=320 \mathrm{~cm}$. Our simulations refer to oscillations with amplitude $A=1.2$ $\mathrm{cm}$ and frequency $\nu=12 \mathrm{~Hz}$. The qualitative picture we discuss does not change if these values are changed.

The dynamics of the system is determined by the amplitude and the frequency of oscillation, and by the grain properties: size, mass and area fractions. These are defined as

$$
\Phi_{l}=N_{l} \frac{\pi}{4 L_{x} L_{y}} \overline{D_{l}^{2}}, \quad \Phi_{h}=N_{h} \frac{\pi}{4 L_{x} L_{y}} D_{h}^{2},
$$

where $\overline{D_{l}^{2}}$ is the mean value of the square of small grains diameter $\left(\overline{D_{l}^{2}} \neq D_{l}^{2}\right.$ when we considering polydisperse light grains), and $N_{h}\left(N_{l}\right)$ is the number of heavy (light) grains.

\section{Dynamics}

The dynamics of a disordered mixture subject to horizontal oscillations can be schematically divided in two steps, stripes formation and stripes coarsening:

- Stripe formation After few oscillations of the tray particles of the same species organize in cluster, which rapidly merge and orientate giving rise to a pattern of stripes perpendicular to the driving direction, as shown in Fig. 3.

- Coarsening On a much longer timescale a coarsening process takes place: stripes of particles of the same kind merge. Consequently the number of stripes decreases and the mean stripe width increases.

This qualitative explanations of the dynamics is formally described by the temporal evolution of the quantity a characteristic length of the system in the $x$ direction, $\xi_{x}$. As usual in coarsening processes this length is defined as:

$$
2 \pi \xi_{x}=\int d k_{x} S_{x}\left(k_{x}\right) d k_{x}
$$

where $S_{x}\left(k_{x}\right)=S\left(k_{x}, 0\right)$ is the structure factor for wave vectors $\vec{k}=\left(k_{x}, 0\right)$ with null $y$ component. Fig. 2 shows the time evolution of $\xi_{x}$ for a mixture of grains of equal size, and area fraction $\Phi_{h}=0.20, \Phi_{l}=0.31$. This is well described by the following functional form

$$
\xi_{x}(t)=a+b \exp (-t / \tau)+c t^{\alpha}
$$



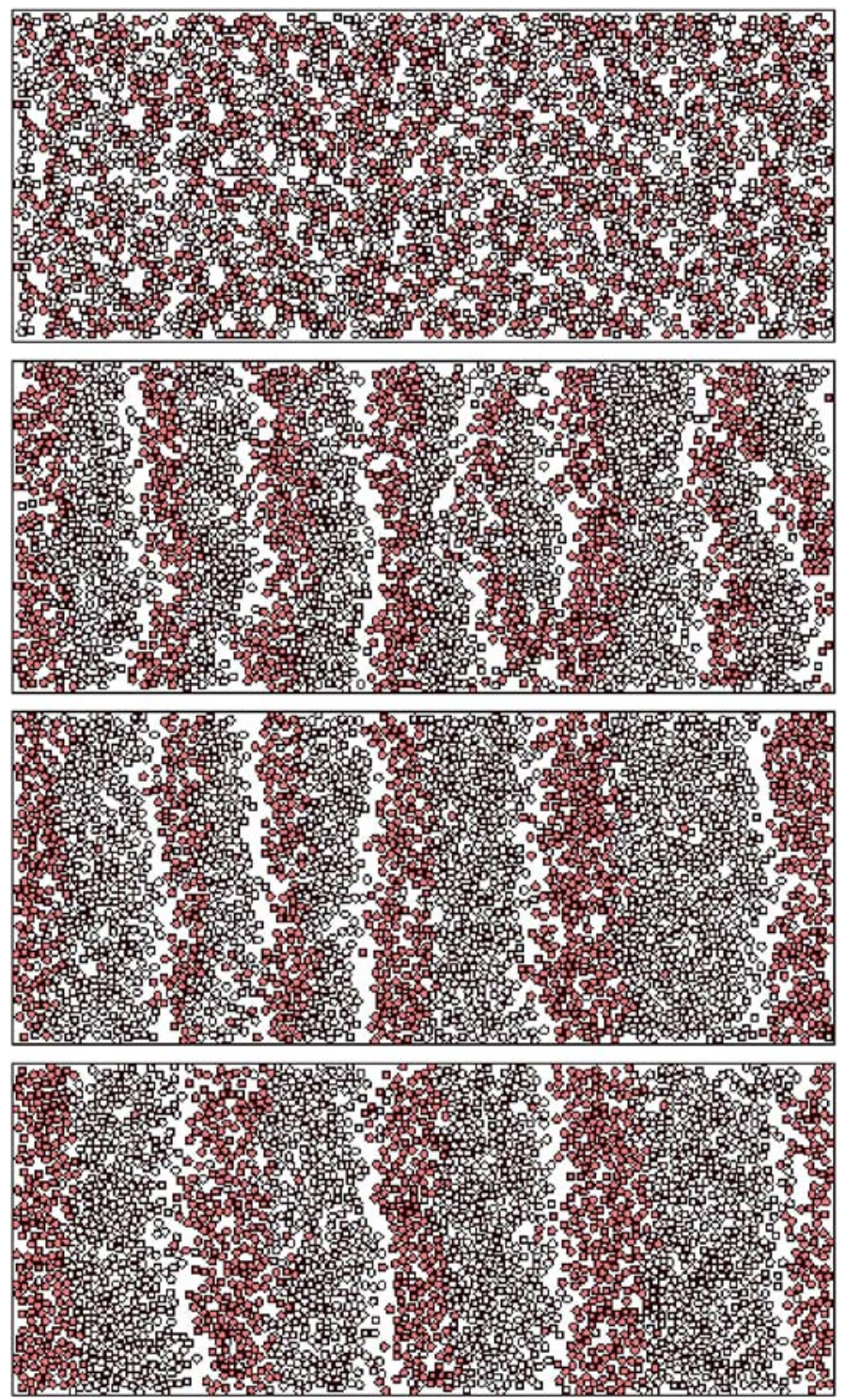

Fig. 1. Evolution of a mixture of equal-sized grains subject to horizontal vibrations. The plots show the state of the system (from top to bottom) after 0, 400, 9800 and 20500 oscillations. Periodic boundary conditions are used in both directions. Note that about 7 stripes are observed after 400 taps, which becomes 4 after 20500 taps. 
which combines an exponential relaxation with lasts approximately $\tau \simeq 175$ oscillations, with a subsequent coarsening process. In this last stage the characteristic length grows with a power law with an exponent $\alpha \simeq 0.25$. A similar growth exponent has been observed in [8]. We discuss now in some more detail the short dynamics (stripes formation) and the long time dynamics, coarsening.

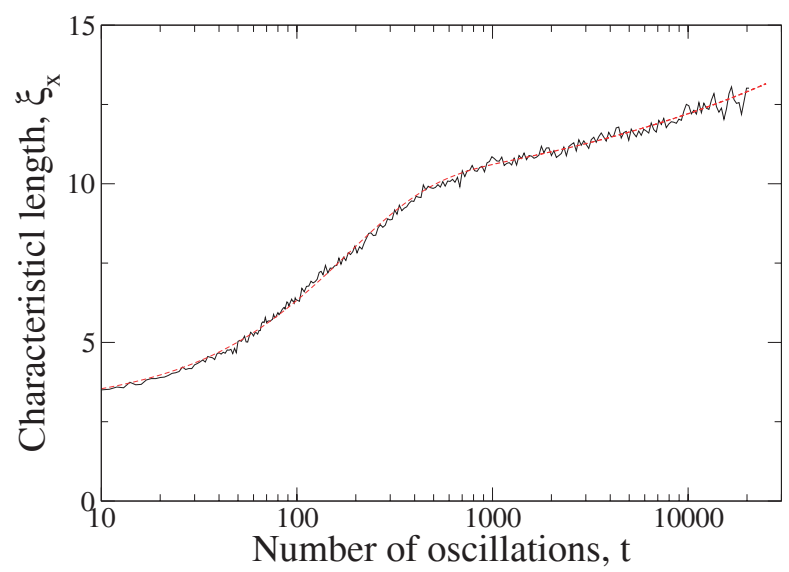

Fig. 2. Temporal evolution of the characteristic length of the pattern. This is well described by eq. 6 (smooth line), which combines an exponential relaxation with a coarsening process.

\subsection{Short-Time Dynamics}

In a previous work we have examined the short time dynamics of the system [12]. The goal was to understand under which conditions stripes form, an what is the dependence of the initial wavelength of the striped pattern on the properties (amplitude and frequency) of the drive. In order to make direct comparisons with the experiments this analysis has been conducted with monodisperse heavy grains, and polydisperse small grains. Under these conditions, which are those studied by T. Mullin and coworkers, the system can be mixed or segregated in stripes. Moreover, if segregation occurs, stripes of the monodisperse species can be either "fluid" or "crystalline".

This behaviour, in the $\left(\phi_{h}, \phi_{l}\right)$ plane, is summarized in the diagram of Fig. 3(a) showing the system "fluid" and "crystal" regions along with their segregation properties, for $\nu=12 \mathrm{~Hz}$ and $A=1.2 \mathrm{~cm}$. Large grains are considered to be in a "fluid" configuration when their radial density distribution function, $g(r)$, shows a first peak at $r=D_{h}$ and a second one at $r=2 D_{h}$, and to be in a "crystal" configuration when a new peak at $r=\sqrt{3} D_{h}$ appears [10]. 

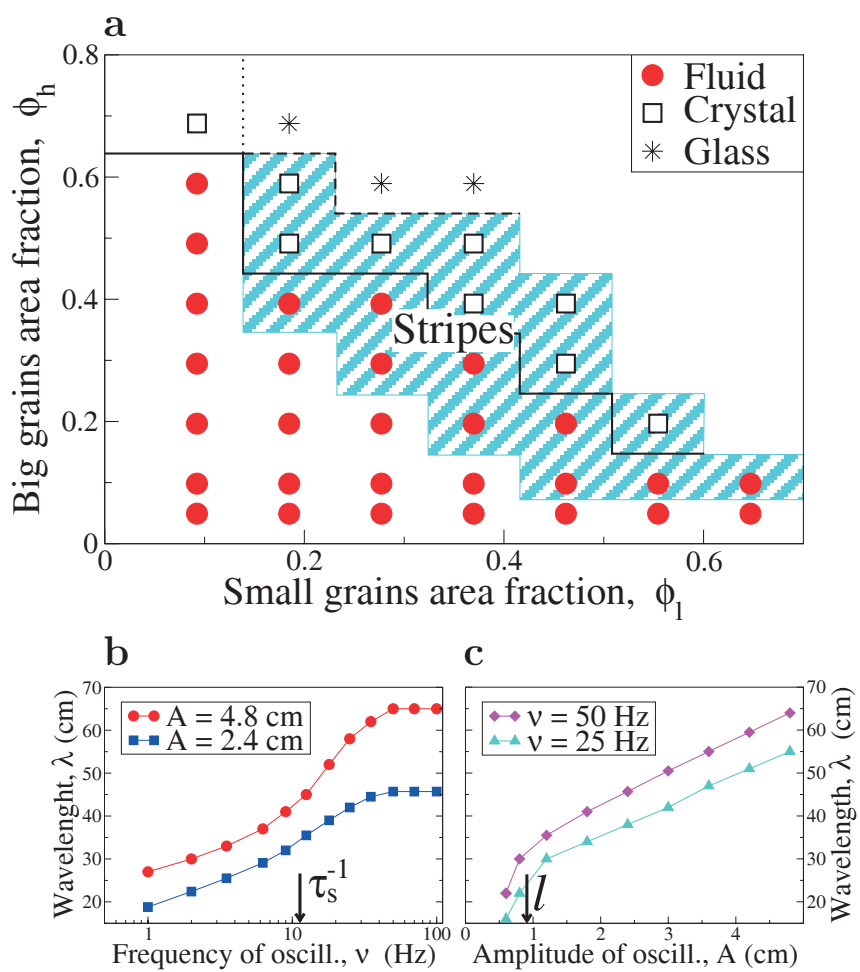

Fig. 3. Panel a Ordering properties of the late stage configurations of the mixture as a function of the area fractions of the two components. The shaded area covers the region where segregation via stripes formation occurs. circles: large grains are in a fluid state. squares: large grains form a crystal. stars: the system appears blocked in a "glassy" disordered configuration (see text). When stripes form their characteristic short time length scale $\lambda$ is a function of the frequency, $\nu$, and of the amplitude, $A$, of the driving oscillations. This is shown, in the case $\phi_{h}=0.30$ and $\phi_{l}=0.28$, in panels $\mathbf{b}$ and $\mathbf{c}$.

The system is in a "glassy" state [19] when on the longest of our observation time scales, the system is still far from stationarity.

Fig. 3(a) shows that grains at small concentrations are mixed and in a fluid state. Segregation via stripes formation appears at higher concentrations. At even higher concentrations, large grains form stripes with a crystalline order, as smaller grains are always fluid for their polydispersity. Finally, at very high area fractions, the system is blocked in its starting disordered configuration ("glassy" region). For instance, by increasing $\phi_{l}$ at a fixed value of $\phi_{h}$ (say $\phi_{h} \simeq 0.174$ ), we observe first a transition from a mixed fluid state to a segregated striped fluid and then a transition where the the monodisperse phase crystallize. The experiments of [10], where $\phi_{h} \simeq 0.174$, show the very same transitions found here at locations differing by a $10 \%$. 
In the case $\phi_{h}=0.30$ and $\phi_{l}=0.28$, where stripes form, we describe their dependence on the dynamics control parameters in Figs. 3(b) and 3(c), showing that the length scale $\lambda=d_{x} / n$, with $d_{x}$ later dimension of the tray and $n$ number of stripes, increases as a function of the shaking frequency, $\nu$, and of the amplitude $A$. These results are to be compared, for instance, with those found in liquid-sand mixtures under oscillating flow: as we will discuss in the next section, in fact, is it possible that in the investigated system stripes form as a result of a dynamical instability of Kelvin-Helmholtz type, the same instability responsible for ripples formation in liquid-sand mixtures. While our results show that the wavelength depends both on the amplitude and the frequency of oscillations, in liquid sand mixture the wavelength depends on the amplitude of oscillation, but not on its frequency [20].

In our system the dependence on $\nu$ can be schematically understood by comparison with the characteristic time scales $\tau_{h}=M_{h} / \mu_{b}$ and $\tau_{l}=M_{l} / \mu_{s}$ of the two species (here $\tau_{h}^{-1}=0.28 \mathrm{~Hz}$ and $\tau_{l}^{-1}=11.3 \mathrm{~Hz}$ ): in the limit $\nu \gg \tau_{h}^{-1}, \tau_{l}^{-1}$ grains are not able to follow the tray motion and no sensitivity to $\nu$ is expected, as well as when $\nu \ll \tau_{l}^{-1}$ since the grains move with the tray. Analogously, the dependence on $A$ is expected to be substantial when $A$ is at least of the order of the mean grains separation length, $l=\left(4 \phi_{h} / \pi D_{h}^{2}+\right.$ $\left.4 \phi_{l} / \pi D_{l}^{2}\right)^{-1 / 2}$, since under this condition grains strongly interact.

\subsection{Long-Time Dynamics}

The long time dynamics of the system is characterized by a coarsening process in which the characteristic length of the system increases as a power law, $\xi_{x}(t) \propto t^{\alpha}$, with an exponent $\alpha \simeq 1 / 4$, as Fig. 2 shows.

The term 'coarsening' is usually referred to describe the out-of equilibrium dynamics of a binary mixture (or of a magnetic system) whose temperature is quenched from a high value to a value $T_{q}$ which is below the coexistence curve. At $T_{q}$ the stable state is made of two coexisting regions: one region is rich in one component of the binary mixture (has positive magnetization), while the other region is rich in the second component of the mixture (has negative magnetization). Therefore the system, which shortly after the quench is still in a mixed state, spontaneously segregate forming growing domains rich in one species or the other. These characteristic size of these domains grow in time with a power law with an exponent which is $1 / 4$ for the case of conserved order parameter (the binary mixture case), $1 / 3$ if the order parameter is not conserved (magnetic system) (for a comprehensive review see [21]).

The long-time dynamics of our system exhibits a phenomenology which closely resemble that of a thermal system undergoing coarsening. However it is worth noting that (at the moment) this coarsening process cannot be interpreted like the phase separation of a binary mixture quenched below the critical point. For instance, it is difficult to introduce a temperature in our system. If we try to define the temperature as the velocity fluctuations, in fact, we end up in a confusing situations: for each species the fluctations of the $x$ and 
of the $y$ component of the velocities are different (as if the temperature was a vector), the temperature of the two components are different, and the velocity distributions are not Maxwellian. At the present stage of understanding the notion of temperature and that of stable phase for the investigated system appear to be meaningless, and the coarsening process must be seen as induced by the forcing.

In this respect one may try to devise a simple model to understand the origin of the growth exponent $\alpha$. The idea is to model the fluctuations of the width of the stripes, and to consider that two stripes merge if in contact $[8,22]$. Assuming that all of the trips have the same width in order for two stripes to merge the need to fluctuate of a distance of order $\xi_{x}$. Therefore we have:

$$
\frac{\partial \xi_{x}}{\partial t} \propto n \cdot d_{y} \cdot P\left(\xi_{x}\right)
$$

where $n=d_{x} /\left(2 \xi_{x}\right)$ is the number of stripes, $P(x)$ is the probability that a point of a stripe fluctuates of a distance $x$, and $d_{y}$ the $y$ length of the tray. In order to estimate $P(x)$ we assume that each point of a stripe makes a random walk in the horizontal direction as a consequence of the various collisions; $P(x)$ is proportional to the time $\tau(x)$ we have to wait for a point to be displaced by $x: P(x) \propto \tau(x) \propto 1 / x^{2}$. With this assumption:

$$
\frac{\partial \xi_{x}}{\partial t} \propto \frac{1}{2} d_{x} d_{y} \frac{1}{\xi_{x}^{3}},
$$

and therefore $\xi_{x}(t) \propto t^{1 / 4}$.

\section{Dynamical Instability}

We have seen so far that a disordered granular mixture subject to horizontal oscillations segregates via the formation of stripes. Here we discuss the evolution of the same system when the initial state is not disordered. On the contrary the two species are placed in two stripes parallel to the driving direction, as show in 4 . The solution of the equation of motion of a grain of mass $M$ interacting with the oscillating tray via a viscous force regulated by the coefficient of friction $\mu$ is

$$
x(t)=-\frac{A}{1+\tau^{2} \nu^{2}}[\cos (\nu t)+\tau \nu \sin (\nu t)],
$$

where $\tau=M / \mu$. In our system the two species, having different relaxation times $\tau_{h}=M_{h} / \mu_{h}=3.57 \mathrm{~s}$ and $\tau_{l}=M_{l} / \mu_{l}=0.09 \mathrm{~s}$, are thus forced to oscillate with different amplitudes and different phases. In the configuration shown in 4 (upper panel) one one may expect the two species to oscillate independently (following equation 9 with different relaxation times), and the initial configuration to be a stable one. But this is not the case. The oscillatory 


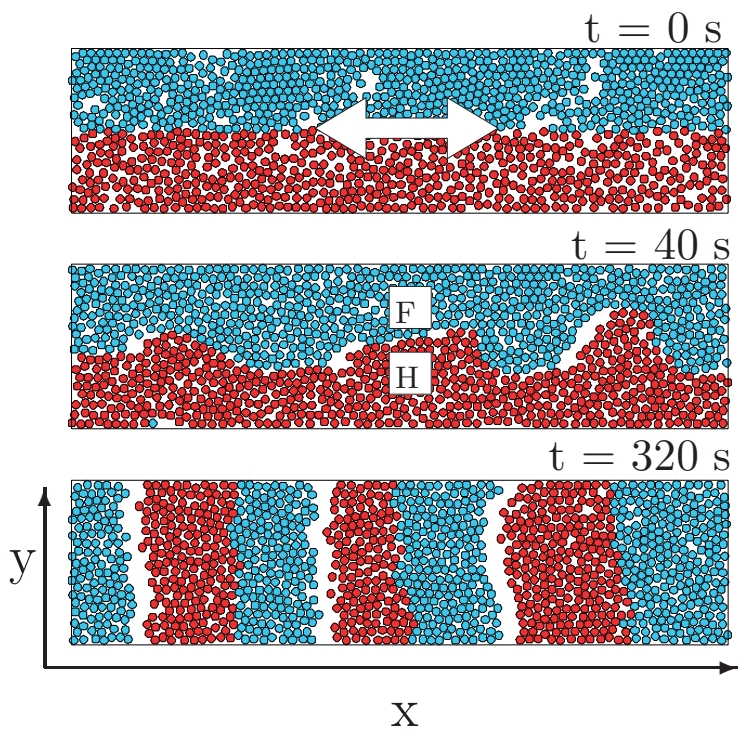

Fig. 4. Evolution of a binary mixture of disks placed on a tray oscillating along the $x$ direction. Here we consider the case in which the diameters of particles of different species are equal. The pictures shows only $1 / 4$ of the system length, which is of $320 D_{b}$. The initial state $(\mathrm{t}=0 \mathrm{~s})$ is made of two stripes of particles of different species parallel to the driving direction. As times goes on the flat interface between the two species evolves via the formation of a sine-like modulation $(\mathrm{t}=40 \mathrm{~s})$. Finally, the wavy interface between grains of different species breaks leading to the formation of a striped pattern as seen before $(\mathrm{t}=320 \mathrm{~s})$.

motion of the tray induces an oscillating shear velocity at the interface between the two species which causes the interface to evolve via the formation of a modulation with a sine-like shape. As times goes on the amplitude of the modulation grows until it breaks giving rise to the striped pattern seen before.

The mechanism responsible for the evolution of the pattern is understood by considering Fig. 4 at $\mathrm{t}=40 \mathrm{~s}$, and by making use of fluid-dynamics considerations. Here ' $\mathrm{F}$ ' and ' $\mathrm{H}$ ' mark regions in which the horizontal motion of grains of a given species is free, or hindered by the presence of grains of the other species. By virtue of Bernoulli's law the pressure in ' $\mathrm{F}$ ' is smaller than the pressure in ' $\mathrm{H}$ ', implying a growth of the perturbed interface. This sets-up a mechanism with a positive feedback, which leads to the formation of the striped pattern. At the moment a closer connection between the investigated system and instability in fluids appears difficult. For instance one is tempted to study the instability via a generalization of the Kelvin-Helmholtz instability (which is a well-known fluid mechanical instability observed when there is a constant shear velocity between two fluid flowing one past the the other $[23,24])$ to the case where the shear velocity between the two fluids 
oscillates in time. However the Kelvin-Helmholtz instability is investigated introducing a typical lengthscale of the system, the capillary length, a combination of gravity and surface tension: in the investigated system gravity plays no role, and there is no surface tension.

A dynamical instability similar to the one of Fig. 4 has been observed both in two fluid systems [25] and in liquid sand mixtures [26]. In these cases, however, gravity stabilizes the interface in a wavy like configuration, and stripes perpendicular to the driving direction are not observed.

\section{Conclusion}

We conclude by shortly discussing the mechanisms which have been proposed in order to explain the observed segregation process. Originally the phenomenology was attributed to the depletion potential [9], a form of interaction well known in colloidal systems [27]. Two big spheres immersed in bath of smaller ones are subject to an effective potential, due to an entropic effect (the clustering of big spheres increases the free space available to the smaller ones, and consequently the entropy of the system), which is attractive at small distances. This attractive interaction is used to explain the phase separation of the system. The anisotropy of the drive, in turn, is used to explain why the phase separation manifests via the formation of stripes [9]. However in order for the depletion potential to exists it is necessary that the mixture is made of particles of different size. Since we have observed segregation also in the case of equal-sized particles, we can rule out the depletion potential as a possible explanation of the observed phenomenology (in ref. [13] we show that the depletion potential does not explain segregation even when the two species have different size).

Another mechanism responsible for segregation, the 'differential drag', has been proposed in $[10,28,29]$. Shortly, the authors suggest that since the two species are forced to oscillate with different amplitudes and phases (see eq. 9) there is an effective repulsion between particles of the different species, which is responsible for the observed phenomenology. While it is certainly true that such a repulsion exists and could possibly play a significant role in order to explain the observed phenomenology, we note here that the 'equilibrium' configuration of the system, that is the one that minimize this repulsion, is made of stripes parallel to driving direction, and not of perpendicular stripes as observed. When the stripes are parallel to the driving direction, in fact, particles of different species never interact, while they interact if the stripes are perpendicular to the driving direction.

Finally, in Ref. [12] we have suggested that the segregation process can be related to instability process previously discussed. However there is no direct evidence that this is instability is responsible for the segregation, and it could be that the segregation process and the instability share a common yet unknown microscopic origin. 
In conclusion, even though the overall phenomenology of the segregation process of a granular mixture subject to horizontal oscillations is clear, the microscopic origin of the observed phenomenology is still obscure.

\section{References}

1. A. Kudrolli, Reports on Progress in Physics 67, 209 (2004).

2. A. Rosato, K.J. Strandburg, F. Prinz and R.H. Swendsen, Phys. Rev. Lett. 58, 1038 (1987).

3. J. Bridgewater, Powder Technol. 15, 215 (1976).

4. J.C. Williams, Powder Technol. 15, 245 (1976).

5. T. Shinbrot and Fernando J. Muzzio, Phys. Rev. Lett. 81, 4365 (1998).

6. J.B. Knight, H.M. Jaeger, and S.R. Nagel, Phys. Rev. Lett. 70, 3728 (1993).

7. M. Tarzia, A. Fierro, M. Nicodemi, and A. Coniglio Phys. Rev. Lett. 93, $198002(2004)$.

8. T. Mullin, Phys. Rev. Lett. 84, 4741 (2000).

9. P.M. Reis and T. Mullin, Phys. Rev. Lett. 89, 244301 (2002).

10. P.M. Reis, G. Ehrhardt, A. Stephenson and T. Mullin, Europhys. Lett. 66, 357 (2004).

11. G. Ehrhardt, A. Stephenson and P.M. Reis, Phys. Rev. E 71, 041301 (2005).

12. M. Pica Ciamarra, and M. Nicodemi and A. Coniglio, Phys. Rev. Lett. 94, 188001 (2005).

13. M. Pica Ciamarra, and M. Nicodemi and A. Coniglio, J. Phys.: Condens. Matter 17, S2549 (2005).

14. M. Pica Ciamarra, and M. Nicodemi and A. Coniglio, in Powders and Grains 2005, edited by R. Gracía-Rojo, H.J. Herrmann and S. McNamara.

15. H.J. Herrmann and S. Luding, Cont. Mech. Thermod. 10, 189-231 (1998).

16. P.A. Cundall and O.D.L. Strack, Geotechnique 29, 47-65 (1979).

17. L.E. Silbert, D. Ertas, G.S. Grest, T.C. Halsey, D. Levine and S.J. Plimpton, Phys Rev E 64, 051302 (2001).

18. R. Mannella, in Stochastic Processes in Physics, Chemistry, and Biology, J.A. Freund and T. Pöshel (ed.s) Springer-Verlag (2000); T. Pöshel and T. Schwager, Computational granular dynamics, Springer (2004).

19. "Unifying concepts in granular media and glasses", (Elsevier Amsterdam, 2004), Edt.s A. Coniglio, A. Fierro, H.J. Herrmann, M. Nicodemi.

20. A. Stegner, J.E. Wesfreid, Phys. Rev. E 60, R3487 (1999).

21. N. Goldenfeld, Lecture on phase transitions and the renormalization group, Addison-Wesley, 1992.

22. P.A. Mulheran, J. Phys. I France 4, 1 (1994).

23. H. Lamb, Hydrodynamics (Cambridge Univ. Press, Cambridge, 1932).

24. S. Chandrasekhar, Hydrodynamic and Hydromagnetic stability. (Clarendon, Oxford, 1961).

25. A.A. Ivanova, V.G. Kozlov and P. Evesque, Fluid Dynamics 36 362, (2002).

26. M.A. Scherer, F. Melo, M. Marder, Phys. of Fluids 11, 58-67 (1999).

27. S. Asakura and F.Oosawa, J. Chem. Phys. 22, 1255 (1954).

28. C.M. Pooley, and J.M. Yeomans, Phys. Rev. Lett. 93, 118001 (2004).

29. P. Sánchez, M.R. Swift, and P.J. King, Phys. Rev. Lett. 93, 184302 (2004). 